\title{
An introduction to
}

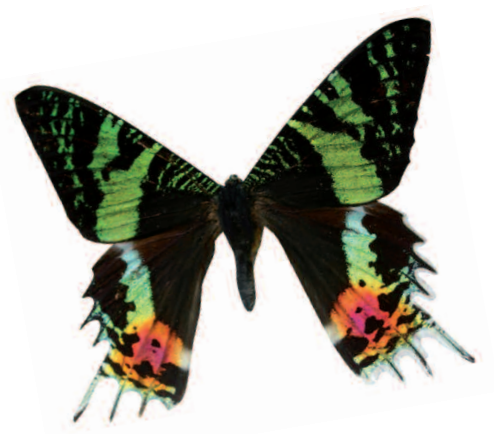

biomimetic

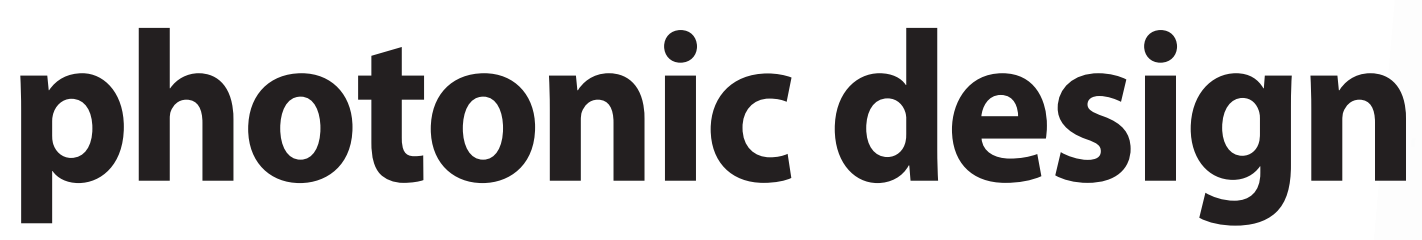

S.M. Luke and P. Vukusic - DOI: 10.1051/epn/2011302

College of Engineering, Mathematics and Physical Sciences, University of Exeter, Stocker Road, Exeter EX4 4QL, UK.

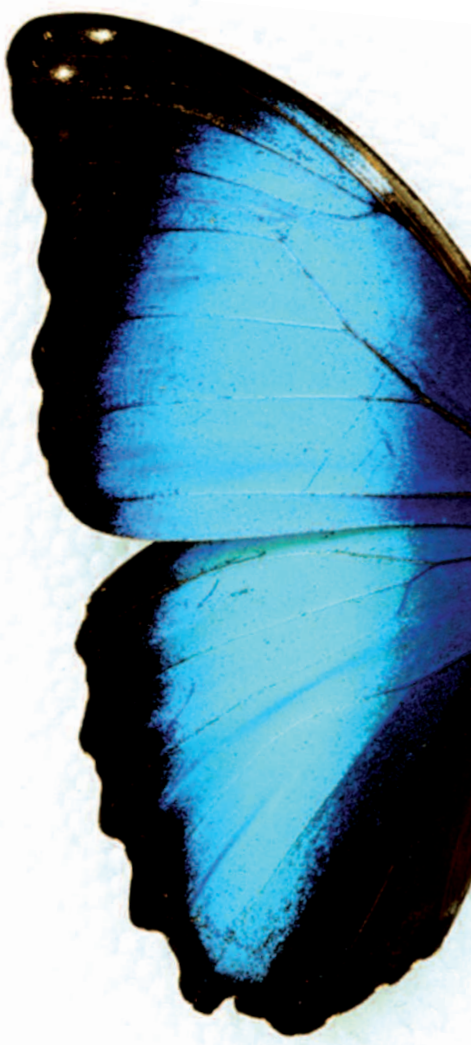

Nature uses a large number of optical phenomena to create a vast array of coloured appearances. The brightest colours and most dazzling optical effects are often those which are created through the use of intricate microstructures that manipulate light. Bio-inspired designs are increasingly leading to improvements in diverse arrays of products for which manipulation of light and appearance is important.

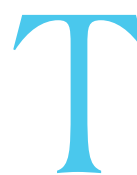

he natural world exhibits many examples of efficient design and specialised functionality. Scientists have long sought inspiration from the natural world; biomimetic design is responsible for many everyday products, for example Velcro. Inspired by the minute hooks on the seeds of the burdock plant, Swiss engineer George de Mestral produced a synthetic mimic which has since found a multitude of applications. Optical biomimetics is a research discipline inspired by optical systems found in nature and by the bright appearances that often result from such systems. Colour in nature can be attributed either to wavelength-selective absorption by chemical pigments, to colour from wavelength-selective reflection from micro-scale structure, or a combination of both. Structural colour can offer ultra-brightness and saturation, as well as offering other interesting properties such as iridescence and polarisation-dependant reflection.
Highly saturated colours, such as those found on the blue wings of many Morpho butterflies, often result from coherent scattering [1]. This arises when light is scattered from periodically-arranged discrete changes in refractive index. A strongly scattered reflection maximum arises at a given wavelength and therefore with a distinctive colour. The colour depends on the periodicity, the refractive indices of the materials and the angles of illumination and observation.

Multilayer structures that offer such periodicity are common in nature. They are often found in brightly coloured beetle species such as the buprestid Chrysochroa raja (shown in figure 1) [2]. In this species a $1.5 \mu \mathrm{m}$ layer on the wing casing surface contains alternating layers of two materials, each with a thickness of approximately $100 \mathrm{~nm}$. The characteristic reflection from this structure is the bright green colour seen at normal incidence. The reflected colour shifts towards blue at higher incident angles, a feature of the iridescent effect (notice in figure 1 where the outer edges of the wing casings appear blue).

The bright blue of many Morpho butterfly wings is the result of a discrete multilayer structure [1]. Each wing is imbricated with a layer of scales which feature prominent structural ridges. A series of lamellae protrude from each ridge giving it 'Christmas tree'like profile in cross-section (see figure 1). The periodicity of the discrete multilayer and the large number of lamellae result in an intense blue reflection (up to $80 \%$ ) that can be seen 'from a quarter mile off' [1]. Long-range signalling is the likely function of such colouration. 


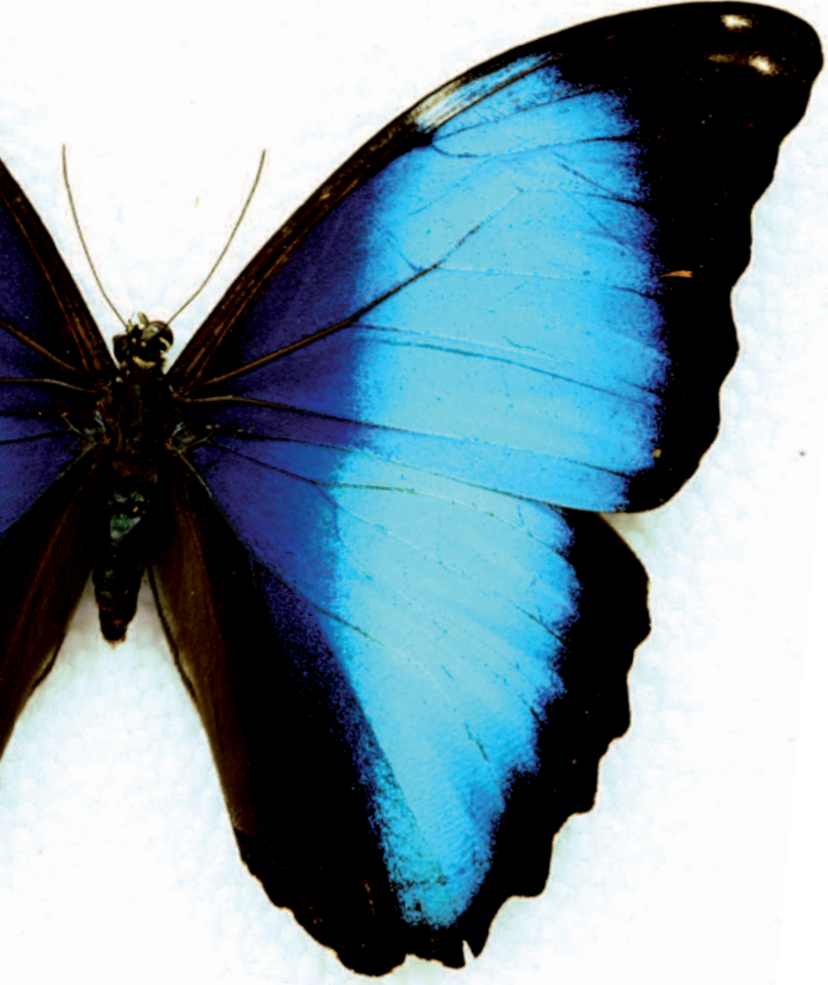

\section{Photonic crystals in nature}

The term 'photonic crystal' applies to any ordered sub-wavelength structure that affects the propagation of light, such as the 1D periodic systems described above. The term is more often applied, however, to 3D ordered crystals. The weevil Eupholus magnificus is one example of many 3D photonic crystal systems found in nature. E. magnificus exhibits a chitin matrix within its scales which is periodically perforated with air holes (figure 2). This 3D structure acts in a similar manner to a $1 \mathrm{D}$ structure, a coloured reflection results from coherent scattering from the periodically-arranged scattering centres that make up the crystal [3].

Each scale comprises discrete domains; these are regions where the orientation of the same $3 \mathrm{D}$ crystalline structure is changed. The characteristic reflectance from each domain is thus slightly different. As the domain size is below the resolution limit of the human eye, the macroscopically perceived colour is an additive mix of all of the domain colours. Rotation of the viewing angle, therefore, has little effect on the perceived colour; the domained structure creates angle-independent colour [3].

\section{Bright whiteness of highly disordered systems}

The highly periodic structures described so far are responsible for bright saturated colours. The converse of this is a highly disordered system which results in bright whiteness. Periodic structures produce relatively saturated colours. Aperiodic structures, however, produce white appearances since all wavelengths are scattered approximately equally. Such disordered systems have been identified both in butterflies (Pieris rapae) and in several species of beetle. Both Cyphochilus (figure 2) and Lepidiota stigma have a coating of white scales which contain a highly disordered filamentary structure [4]. Structural analysis confirms the absence of periodicity and indicates that the system is highly optimised for maximum optical scatter and thus whiteness and brightness. The whiteness of these scales, those of Cyphochilus in particular, compares favourably with those measured from many synthetic materials. (a)

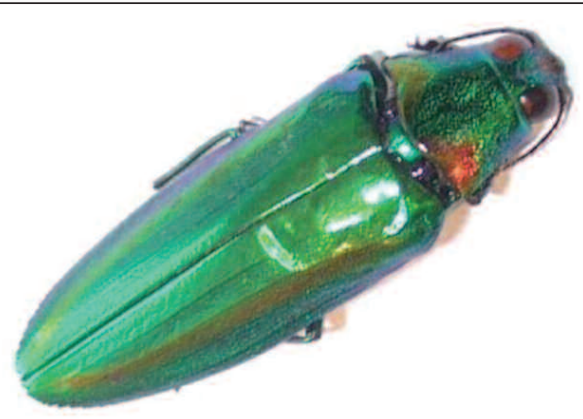

(b)

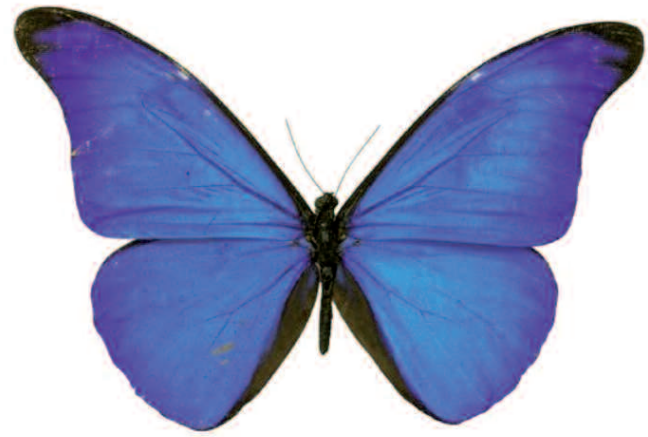

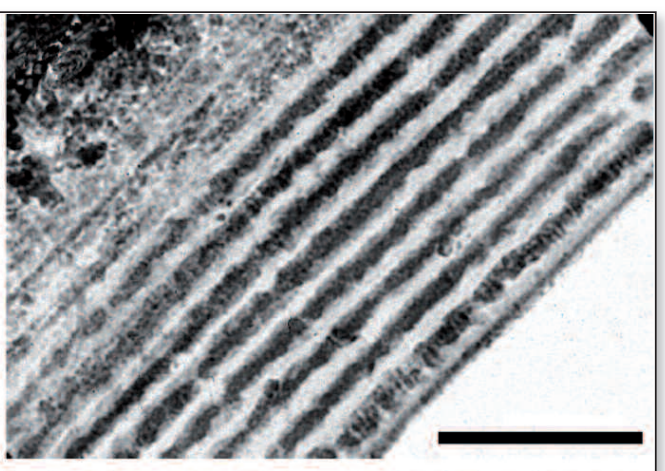

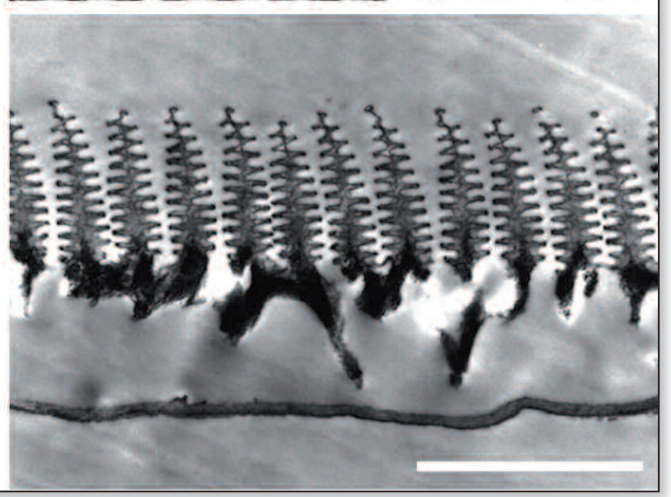

$\triangle$ FIG. 1: Both Chrysochroa raja (a) and many species of Morpho butterfly

(b) derive their bright colours through constructive interference of light reflected from their periodic microstructured surfaces (as seen in the accompanying structural images). Scale bars (a) $900 \mathrm{~nm}$ (b) $1.8 \mu \mathrm{m}$. (Beetle image in (a) courtesy of Dr. Joseph Noyes). 


\section{Mimicking nature's design -}

Structurally coloured fabric and cosmetics Designing and developing applications for optical functions through bio-inspiration has gained momentum in the last decade. Lepidoptera, which display some of the brightest colours, have been at the forefront of this inspiration. The brilliant blue Morpho butterfly has provided inspiration for two diverse products. L'Oreal has pioneered bio-optical inspiration in the cosmetics industry. By mimicking the manner in which light interacts with Morpho scales, but using synthetic materials to form periodic nanostructures, L'Oreal has created a photonic cosmetic product which contains no chemical pigments (see figure 3 ).

Morpho species were also arguably the inspiration for a structurally coloured fabric known as Morphotex [5]. The fibre contains no dye but produces its colour through coherent scattering. 61 layers of polyester and nylon are laminated together into a multilayer structure. Altering the layer periodicity produces a fibre which is either red, blue, green or violet in colour. The fabric contains no dye, which has both economic and environmental benefits. The structural nature of the colour means that the fabric exhibits unlimited lightfastness. This makes it a desirable product for applications in the fashion industry (see figure 3) and automotive or domestic furnishing textiles.

A further bio-inspired fabric technology which is in development is an 'optical shield' coating, inspired by the Cyphochilus structure [6]. The beetle's disordered filamentary structure reflects visible and UV wavelength light with remarkable efficiency. The structure, therefore, acts as an extremely effective and efficient optical 'shield' for visible and UV wavelengths.

The technology for this comprises electro-spun plastic nanofiber webs [6]. Optimum whiteness is achieved with nanofibers that have an average diameter of $250 \mathrm{~nm}$; this is similar to the filament diameters of Cyphochilus. In this application it is the optical shielding that the structure offers that is important. Polyurethane (PU) foam is widely used in the clothing industry but suffers from unsightly yellowing upon UV irradiation. These nanofiber web coating layers provide good UV protection and can be sprayed directly onto the PU foam product, thereby simplifying its manufacturing process.

\section{Bio-inspired improvements in paper industry}

The paper industry is another example where bio-inspiration is being implemented to improve a technology [7]. In this industry whiteness, brightness and opacity are the key factors when characterising a product. Brightness measurements indicate that the Cyphochilus structure is more efficient than conventional claybased paper coatings. Ultra-high efficiency bio-inspired coatings could conceivably reduce coating material requirements with no detrimental optical effects.

Optimisation of the beetle structure's filling fraction, scattering centre size and spacing appears to underpin its exceptional whiteness. Optimisation of the paper coating layer in a similar manner would see an
- FIG. 2:

(a) The bright green coloured stripes of Eupho-

Ius magnificus result from interference of light reflected from parallel planes in its 3D photonic crystal structure.

(b) A highly disordered filamentary structure gives Cyphochilus a dis tinctive bright white appear ance. Scale bars: (a) $2 \mu \mathrm{m}$ (b) $3 \mu \mathrm{m}$.
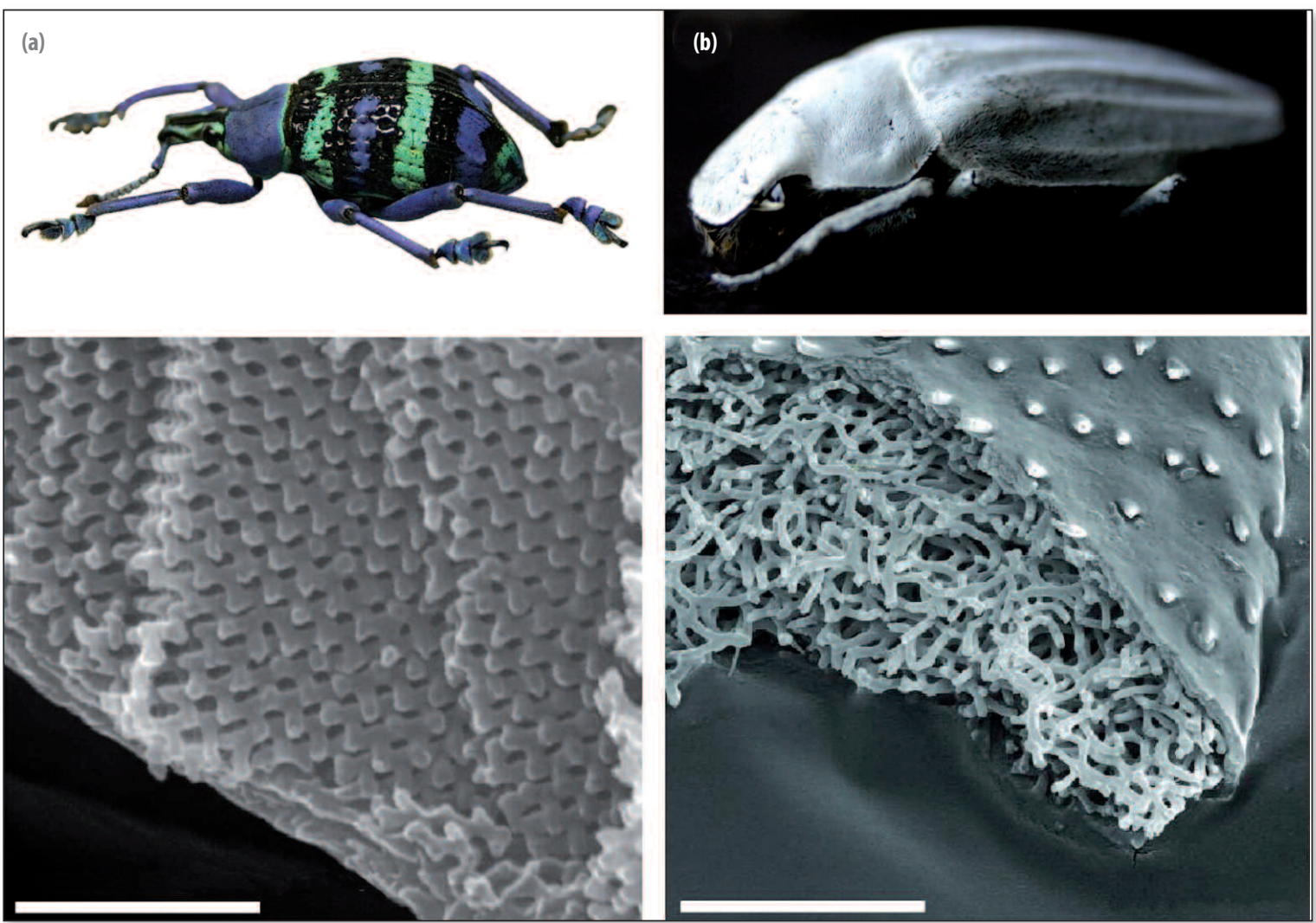
